\title{
Fitting ideals of class groups in a $\mathbb{Z}_{p}$-extension
}

\author{
by
}

\author{
Pietro Cornacchia (Asti)
}

1. Introduction. In Section 2 of this paper we prove a purely algebraic result on the structure of torsion Iwasawa modules. Let $p$ be a prime number, let $\Gamma$ be a profinite group isomorphic to $\mathbb{Z}_{p}$, and let $\chi: \Delta \rightarrow \overline{\mathbb{Q}}_{p}^{*}$ be a $p$-adic character of a finite abelian group $\Delta$ of order prime to $p$. We denote by $\mathcal{O}_{\chi}$ the discrete valuation ring $\mathbb{Z}_{p}(\chi)$. Let $\Lambda=\mathcal{O}_{\chi}[[\Gamma]] \cong \mathcal{O}_{\chi}[[T]]$ be the Iwasawa algebra. The ring $\Lambda$ is a local regular ring of dimension 2 , with maximal ideal $\mathfrak{m}=(p, T)$. For each $n \in \mathbb{N}$, we set $\omega_{n}=(1+T)^{p^{n}}-1 \in \Lambda$. For any $\Lambda$-module $M$, we write $M_{n}$ for $M / \omega_{n} M$. We denote by $G_{n}$ the unique quotient of $\Gamma$ which is cyclic of order $p^{n}$ and by $G_{m, n}$ the subgroup of $G_{m}$ of order $p^{m-n}$. The symbol $\widehat{H}$ denotes Tate cohomology groups. We denote by Fit $_{R}$ the Fitting ideals over a ring $R$. We prove the following

TheOREM 1. Let $M$ be a $\Lambda$-module which is finitely generated as a $\mathbb{Z}_{p^{-}}$ module. Suppose that $M_{n}$ has finite order for all n. Let $T(M)$ be the torsion $\mathbb{Z}_{p}$-submodule of $M$, and let $\operatorname{char} M$ be its characteristic ideal. We have

(1) $T(M) \cong \widehat{H}^{i}\left(G_{m, n}, M_{m}\right)$ for any $i \in \mathbb{Z}$, provided that $n$ and $m-n$ are sufficiently large,

(2) $\operatorname{Fit}_{\Lambda} M=\operatorname{Fit}_{\Lambda} T(M) \cdot \operatorname{char} M$,

(3) $\# M_{n}=\# T(M)_{n} \cdot \#\left(\Lambda_{n} /(\operatorname{char} M) \Lambda_{n}\right)$.

Roughly speaking, the cohomology groups at finite levels determine the $\mathbb{Z}_{p}$-torsion submodule $T(M)$, while the characteristic ideal gives information on the $\mathbb{Z}_{p}$-free part of $M$, that is, the quotient $M / T(M)$. This information is enough to determine the Fitting ideal of $M$ and the orders of the modules $M_{n}$. Observe that the condition that $M$ is finitely generated as a $\mathbb{Z}_{p}$-module is equivalent to saying that its Iwasawa $\mu$-invariant is zero. This is often the case for $\Lambda$-modules arising from arithmetic applications.

1991 Mathematics Subject Classification: 11R18, 11R23, 11R29.

The present studies were supported in part by a CNR grant. 
In Section 3 we apply Theorem 1 to an arithmetic situation. Let $K$ be a totally real abelian number field with Galois group $\Delta$ such that $p \nmid \# \Delta$. Suppose that there is only one prime $\wp$ of $K$ above $p$. If $p=2$, we also require that $K$ has prime power conductor. Let $\left\{K_{n}\right\}$ be the cyclotomic $\mathbb{Z}_{p}$-extension of $K$. Let $\chi$ be a nontrivial $p$-adic character $\chi: \Delta \rightarrow \overline{\mathbb{Q}}_{p}^{*}$. For any $\mathbb{Z}[\Delta]$ module $D$, we define its $\chi$-part $\left(D \otimes_{\mathbb{Z}} \mathbb{Z}_{p}\right) \otimes_{\mathbb{Z}_{p}[\Delta]} \mathcal{O}_{\chi}$. For more information on $\chi$-parts, see for example [3]. Let $A_{n}$ and $B_{n}$ denote respectively the $\chi$-parts of the ideal class group and of the group of units modulo cyclotomic units of the field $K_{n}$. The group of cyclotomic units will be defined in Section 3. Let $\Lambda_{n} \cong$ $\Lambda / \omega_{n} \Lambda \cong \mathcal{O}_{\chi}\left[\operatorname{Gal}\left(K_{n} / K\right)\right]$. M. Ozaki $[15,16]$ already studied the structure of $A_{n}$ and $B_{n}$ as $\Lambda_{n}$-modules. We prove an equality of Fitting ideals.

TheOREM 2. Fit $A_{n} A_{n}=$ Fit $_{\Lambda_{n}} B_{n}$ for all $n \in \mathbb{N}$.

In order to prove this we consider two $\Lambda$-modules: the projective limit $A$ of the groups $A_{n}$, and the projective limit $C$ of the Pontryagin duals of the groups $B_{n}$. We take duals, because the module $C$ has functorial properties analogous to those of $A$. We show that both $A$ and $C$ satisfy the hypothesis of Theorem 1. Class field theory allows us to compare the cohomology groups of $A_{n}$ and $B_{n}$. The main conjecture of Iwasawa theory and the theory of adjoints give us that $A$ and $C$ have the same characteristic ideal. Applying Theorem 1(2) and descending at finite levels we obtain our claim. As an immediate application of Theorem 1(3) we show that $\# A_{n}=\# B_{n}$ for all $n$ (Theorem 3). This is a particular case of Theorem 9.2 of [12] (see also Theorem 4.14 of [7]), but the proof in our situation is much simpler. In the last section we make some remarks about the results obtained.

I would like to thank René Schoof for stimulating my interest in this problem and Cornelius Greither for his preprint [8] and for helpful discussions.

2. Fitting ideals of $\Lambda$-modules. In this section, we prove Theorem 1 . In order to proceed to the proof, we review some algebraic facts. Let $F$ be a torsion $\Lambda$-module which is a free finitely generated $\mathbb{Z}_{p}$-module. Since $\Lambda$ is regular of dimension 2, the Auslander-Buchsbaum formula implies that $F$ has projective dimension 1 over $\Lambda$. In particular, we have a resolution

$$
0 \rightarrow \Lambda^{r_{1}} \stackrel{\lambda}{\rightarrow} \Lambda^{r_{2}} \rightarrow F \rightarrow 0 .
$$

In fact every projective $\Lambda$-module is free, because $\Lambda$ is local, and we must also have $r_{1}=r_{2}=r$ because $F$ is $\Lambda$-torsion. The $\Lambda$-Fitting ideal of $F$ is the principal ideal generated by the determinant of the matrix associated with $\lambda$. For all height one prime ideals $\wp$ of $\Lambda$, the localization $\Lambda_{\wp}$ is a principal ideal domain and $\left(\text { Fit }_{\Lambda} F\right)_{\wp}=$ Fit $_{\Lambda_{\wp}} F_{\wp}=(\operatorname{char} F)_{\wp}$. The ideals Fit $_{\Lambda} F$ and char $F$ are both principal and their localizations at all height 
one prime ideals of $\Lambda$ coincide. This implies Fit $_{\Lambda} F=\operatorname{char} F$. See also the appendix of [14]. We now need a lemma.

LEMma 1. Let $F$ be a $\Lambda$-module which is a free finitely generated $\mathbb{Z}_{p^{-}}$ module. Let $\omega \in \Lambda$ and suppose that the distinguished polynomials associated with char $F$ and $\omega$ have no common zeros. Then $\operatorname{Tor}_{1}^{\Lambda}(\Lambda / \omega \Lambda, F)=0$.

Proof. Consider the short exact sequence induced by multiplication with $\omega$

$$
0 \rightarrow \Lambda \stackrel{\omega}{\rightarrow} \Lambda \rightarrow \Lambda / \omega \Lambda \rightarrow 0
$$

Tensoring with $F$ induces an exact sequence

$$
0 \rightarrow \operatorname{Tor}_{1}^{\Lambda}(\Lambda / \omega \Lambda, F) \rightarrow F \stackrel{\omega}{\rightarrow} F \rightarrow F / \omega F \rightarrow 0 .
$$

Since $\operatorname{Tor}_{1}^{\Lambda}(\Lambda / \omega \Lambda, F)$ can be viewed as a submodule of $F$, it is $\mathbb{Z}_{p}$-free. But since $\operatorname{Tor}_{1}^{\Lambda}(\Lambda / \omega \Lambda, F)$ is killed by both $\omega$ and char $F$, the hypothesis implies that there exists a power of $p$ which annihilates it. Therefore $\operatorname{Tor}_{1}^{\Lambda}(\Lambda / \omega \Lambda, F)=0$ as we wanted to show.

Let now $M$ be as in the theorem. We have a short exact sequence

$$
0 \rightarrow T(M) \rightarrow M \rightarrow F \rightarrow 0
$$

where $F$ is a torsion $\Lambda$-module which is free and finitely generated as a $\mathbb{Z}_{p^{-}}$ module. Let $m \in \mathbb{N}$. Since $M / \omega_{m} M$ has finite order, char $M$ and $\omega_{m}$ have no common zeros. Therefore the above lemma applies to $F$. In particular, tensoring (1) with $\Lambda / \omega_{m} \Lambda$ we get a free $\mathcal{O}_{\chi}\left[G_{m}\right]$ resolution of $F_{m}$, which shows that $F_{m}$ is $G_{m, n}$-cohomologically trivial, for all $n \leq m$. We now tensor (2) by $\Lambda / \omega_{m} \Lambda$. Using Lemma 1 we obtain

$$
0 \rightarrow T(M)_{m} \rightarrow M_{m} \rightarrow F_{m} \rightarrow 0 .
$$

Taking cohomology we get

$$
\widehat{H}^{i}\left(G_{m, n}, M_{m}\right) \cong \widehat{H}^{i}\left(G_{m, n}, T(M)_{m}\right)
$$

for all $m \geq n$ and $i \in \mathbb{Z}$. Since $M$ is finitely generated as a $\mathbb{Z}_{p}$-module, the torsion module $T(M)$ has finite order. This implies that $\Gamma^{p^{n}}$ acts trivially on $T(M)$, for sufficiently large $n$. Let now $m>n$ be such that the norm map $N_{G_{m, n}}$ (sum of the elements of $G_{m, n}$ ) kills $T(M)$. For example, it is enough that $p^{m-n}$ is larger than \#T(M). For such values of $m$ and $n$, we have $\widehat{H}^{i}\left(G_{m, n}, T(M)_{m}\right) \cong T(M)_{m}$ for all $i \in \mathbb{Z}$. But since $m>n$, we have $T(M)_{m} \cong T(M)$. This, together with (4), implies the first assertion of Theorem 1.

We now prove the second assertion. I am grateful to Cornelius Greither for showing me the following argument. Start with sequence (2) and take a resolution of $F$ as in (1). Take a surjective map $\Lambda^{k} \rightarrow T(M)$, and let $K$ be its kernel. The map $\Lambda^{r} \rightarrow F$ extends to a map $\Lambda^{r} \rightarrow M$ and we get 
a surjective map $\Lambda^{k} \oplus \Lambda^{r} \rightarrow M$ whose kernel will be denoted by $K_{1}$. The snake lemma gives us a commutative diagram with exact rows and columns

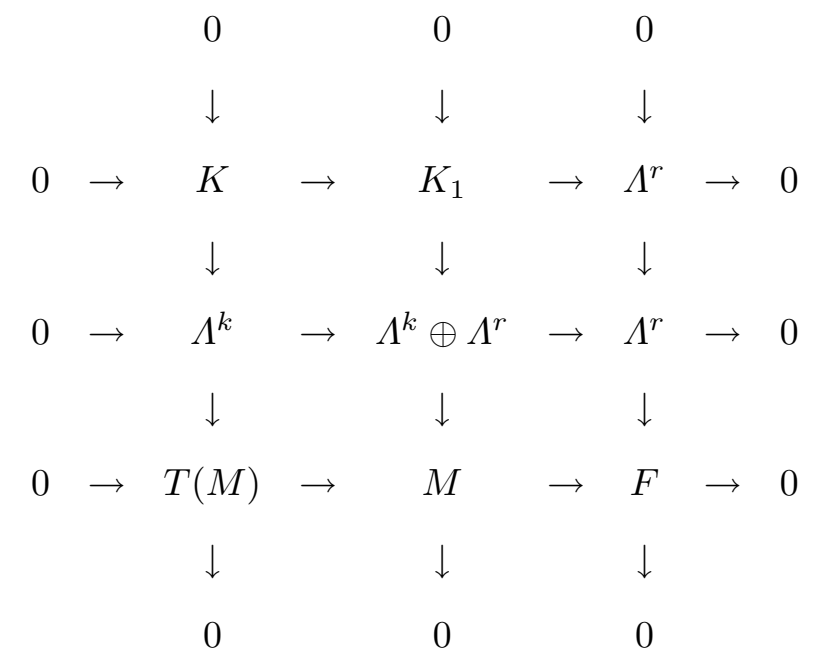

The top row is split and we have $K_{1} \cong K \oplus \Lambda^{r}$. After choosing a set of generators for $K$, the map $\gamma_{1}: K_{1} \rightarrow \Lambda^{k} \oplus \Lambda^{r}$ can be represented by a matrix. The top left block $B$ represents the map $K \rightarrow \Lambda^{k}$, the bottom left block is 0 , and the bottom right block is a square matrix $A$ representing the map $\lambda: \Lambda^{r} \rightarrow \Lambda^{r}$. It is clear that the Fitting ideal of $M$ is generated by the products of $\operatorname{det} A$ times an $r \times r$ minor of $B$. Since $\operatorname{det} A$ generates the Fitting ideal of $F$, and the $r \times r$ minors of $B$ generate the Fitting ideal of $T(M)$, the second assertion of Theorem 1 follows.

To prove the third assertion, consider the exact sequence (3). Since the order is multiplicative in exact sequences, we only need to show that $\# F_{m}=$ $\#\left(\Lambda_{m} /(\operatorname{char} M) \Lambda_{m}\right)$. Since $\operatorname{Fit}_{\Lambda} F=(\operatorname{char} M)$, we have Fit $_{\Lambda_{m}} F_{m}=$ (char $M) \Lambda_{m}$. In particular, it is a principal ideal. From [1, Ch. III, Sec. 9.4, Prop. 6], we have

$$
\# F_{m}=\#\left(\Lambda_{m} / \operatorname{Fit}_{\Lambda_{m}} F_{m}\right)=\#\left(\Lambda_{m} /(\operatorname{char} M) \Lambda_{m}\right) .
$$

The proof is now complete.

3. Ideal class groups and units modulo cyclotomic units. In this section we apply Theorem 1 in order to study the ideal class groups in a cyclotomic $\mathbb{Z}_{p}$-extension. Let $K$ be a real abelian number field. Suppose that $p \nmid[K: \mathbb{Q}]$, and that in the ring of integers of $K$ there is only one prime above $p$. If $p=2$, we require that the conductor of $K$ is a prime power. We denote by $\Delta$ the Galois group of $K$ over $\mathbb{Q}$. Let $\chi: \Delta \rightarrow \overline{\mathbb{Q}}_{p}^{*}$ be a nontrivial $p$-adic character of $\Delta$. Let $K_{n}$ be the unique field of degree $p^{n}$ over $K$ contained in the cyclotomic $\mathbb{Z}_{p}$-extension of $K$. We set $G_{n}=\operatorname{Gal}\left(K_{n} / K\right)$ for all $n \in \mathbb{N}$ and $G_{m, n}=\operatorname{Gal}\left(K_{m} / K_{n}\right)$ for all $m \geq n \geq 0$. 
The group of cyclotomic units for an abelian totally real number field has been defined by W. Sinnott [19, Sec. 4]. Since we also want to deal with the case $p=2$, we slightly modify Sinnott's definition. Let $L$ be any real abelian field. For all $r \in \mathbb{N}$, let $\zeta_{r}$ be a primitive $r$ th root of unity. We define the group $\operatorname{Cyc}\left(E_{r}\right)$ of cyclotomic elements of the field $E_{r}=\mathbb{Q}\left(\zeta_{r}+\zeta_{r}^{-1}\right)$ as the group of units of the form $\mathbb{Z}\left[\operatorname{Gal}\left(E_{r} / \mathbb{Q}\right)\right]\left(1-\zeta_{r}\right)$. Let $L(r)=E_{r} \cap L$. We define the group of cyclotomic units of $L$ as the group generated by the norms $N_{L(r)}^{E_{r}} \operatorname{Cyc}\left(E_{r}\right)$ for all $r \in \mathbb{N}$. The difference with Sinnott's definition is that we take norms of the cyclotomic numbers from the real subfields of the cyclotomic fields instead of the norms from the cyclotomic fields themselves. Our group of cyclotomic units contains Sinnott's one, and the index of the two groups is a power of 2 . In this way, we eliminate extra powers of 2 in the index formulae relating class groups with unit groups.

For all $m \in \mathbb{N}$, we denote by $\mathrm{Cyc}_{m}$ the $\chi$-part of the cyclotomic units of $K_{m}$. Let $f_{m}$ be the conductor of $K_{m}$. Observe that when $p=2$ then $f_{m}$ is divisible by at most two primes. By a theorem of H. Bass (Theorem 8.9 of [20] and the remarks on pp. 260-261) the only relations in the $\chi$-part of the cyclotomic units of $\mathbb{Q}\left(\zeta_{f_{m}}\right)$ are the distribution ones. This is the only place where we use our hypothesis on $K$ in the case $p=2$. Since $p \nmid[K: \mathbb{Q}]$, the $\mathcal{O}_{\chi}$-module $\mathrm{Cyc}_{0}$ is cyclic. Since there is only one prime above $p$ in the ring of integers of $K$, and $\chi$ is not trivial, the group $\mathrm{Cyc}_{m}$ is a free rank one $\mathcal{O}_{\chi}\left[G_{m}\right]$-module. In fact let

$$
\eta_{m}=N_{K_{m}}^{\mathbb{Q}\left(\zeta_{f_{m}}+\zeta_{f_{m}}^{-1}\right)}\left(1-\zeta_{f_{m}}\right)(\chi) .
$$

The distribution relations for cyclotomic units imply that for $k \leq m$, the group $\mathrm{Cyc}_{k}$ is generated up to exponents $\chi\left(\operatorname{Frob}_{p}\right)-1$ by the norm of $\eta_{m}$, where $\operatorname{Frob}_{p} \in \Delta$ is the Frobenius element relative to $p$. Since there is only one prime of $K$ above $p$, the element $\chi\left(\right.$ Frob $\left._{p}\right)-1$ is an $\mathcal{O}_{\chi}$-unit. This means that the map

$$
\mathcal{O}_{\chi}\left[G_{m}\right] \rightarrow \mathrm{Cyc}_{m}
$$

defined by $x \rightarrow \eta_{m}^{x}$ is surjective. Since both $\mathcal{O}_{\chi}\left[G_{m}\right]$ and $\mathrm{Cyc}_{m}$ are free $\mathbb{Z}_{p}$-modules of the same rank, the above map is an isomorphism.

We denote by $A_{n}$ the $\chi$-parts of the ideal class groups of $K_{n}$ and by $B_{n}$ the $\chi$-parts of the groups of units modulo cyclotomic units of the ring of integers of $K_{n}$. For each $n$ we define the group

$$
C_{n}=\operatorname{Hom}_{\mathbb{Z}}\left(B_{n}, \mathbb{Q}_{p} / \mathbb{Z}_{p}\right) .
$$

Observe that $\# B_{n}=\# C_{n}$. We give $C_{n}$ the structure of a $G_{n}$-module by setting $\sigma \varphi(b)=\varphi(\sigma b)$ for all $\sigma \in G_{n}, \varphi \in C_{n}$ and $b \in B_{n}$. For all pairs of natural numbers $m \geq n$ there are norm maps $N_{m, n}: A_{m} \rightarrow A_{n}$ and maps $j_{m, n}: B_{n} \rightarrow B_{m}$. The maps $j_{m, n}$ induce maps $C_{m} \rightarrow C_{n}$. We denote 
by $A$ and by $C$ the inverse limits of the projective systems $\left\{A_{n}\right\}$ and $\left\{C_{n}\right\}$ respectively. These are compact $\Lambda$-modules. We need to know the relation between the $A_{n}$ and $A$ and between the $C_{n}$ and $C$ respectively. Let $\gamma$ be a topological generator of $\Gamma$ (it corresponds to $1+T$ in the identification $\left.\Lambda \cong \mathcal{O}_{\chi}[[T]]\right)$. We set $\gamma_{n}=\gamma^{p^{n}}$.

Proposition 1. For all $n$, we have $A_{n} \cong A / A^{1-\gamma_{n}}$.

This is a classical result in Iwasawa theory. One uses the fact that in $K$ there is only one prime above $p$ and that this prime is totally ramified in the cyclotomic $\mathbb{Z}_{p}$-extension of $K$. See [20, Lemma 13.15].

We have a similar result for the groups $C_{n}$ :

Proposition 2. For all $n$, we have $C_{n} \cong C / C^{1-\gamma_{n}}$.

Proof. Let $m$ be any integer with $m \geq n$, and let $\mathcal{O}_{m}^{*}$ be the $\chi$-part of the units of $K_{m}$. We have a short exact sequence

$$
0 \rightarrow \mathrm{Cyc}_{m} \rightarrow \mathcal{O}_{m}^{*} \rightarrow B_{m} \rightarrow 0 .
$$

Since $\mathrm{Cyc}_{m} \cong \mathcal{O}_{\chi}\left[G_{m}\right]$, we get $H^{1}\left(G_{m, n}, \mathrm{Cyc}_{m}\right) \cong 0$. Therefore after taking $G_{m, n}$-invariants, (5) stays exact and we get $B_{n} \cong B_{m}^{G_{m, n}}$. Let $\sigma_{m, n}$ be a generator for $G_{m, n}$. We have a short exact sequence

$$
0 \rightarrow B_{n} \rightarrow B_{m} \stackrel{1-\sigma_{m, n}}{\longrightarrow} B_{m}^{1-\sigma_{m, n}} \rightarrow 0 .
$$

We apply the contravariant exact functor $\operatorname{Hom}\left(-, \mathbb{Q}_{p} / \mathbb{Z}_{p}\right)$ and get

$$
0 \rightarrow \operatorname{Hom}\left(B_{m}^{1-\sigma_{m, n}}, \mathbb{Q}_{p} / \mathbb{Z}_{p}\right) \rightarrow C_{m} \rightarrow C_{n} \rightarrow 0 .
$$

Now observe that $\operatorname{Hom}\left(B_{m}^{1-\sigma_{m, n}}, \mathbb{Q}_{p} / \mathbb{Z}_{p}\right) \cong C_{m}^{1-\sigma_{m, n}}$. This follows by dualizing the injection $B_{m}^{1-\sigma_{m, n}} \rightarrow B_{m}$. We obtain the exact sequence

$$
0 \rightarrow C_{m}^{1-\sigma_{m, n}} \rightarrow C_{m} \rightarrow C_{n} \rightarrow 0 .
$$

We take the inverse limit with respect to $m$ in the above sequence. Since the inverse system $C_{m}$ is surjective, the above exact sequence stays exact in the limit. From the compactness of $C$, we see that the inverse limit of $C_{m}^{1-\sigma_{m, n}}$ is exactly $C^{1-\gamma_{n}}$. We finally get

$$
0 \rightarrow C^{1-\gamma_{n}} \rightarrow C \rightarrow C_{n} \rightarrow 0 .
$$

This concludes the proof.

The analogy of Proposition 2 with Proposition 1 is the reason why we work with the groups $C_{n}$ instead of $B_{n}$. Observe that by the theorem of Ferrero-Washington $A$ is a finitely generated $\mathbb{Z}_{p}$-module. By Proposition 2 together with Nakayama's lemma, we deduce that $C$ is a finitely generated $\Lambda$-module. Combining this with Theorems 4.1 and 6.1 of [19] it follows that 
the $\mu$ invariant of $C$ is zero. Therefore $C$ is finitely generated over $\mathbb{Z}_{p}$ too, and is a torsion $\Lambda$-module. Therefore we can apply Theorem 1 to $A$ and $C$.

Proposition 3. $T(C) \cong \operatorname{Hom}\left(T(A), \mathbb{Q}_{p} / \mathbb{Z}_{p}\right)$.

Proof. Let $m \geq n \geq 0$. By Theorem 1 , we are reduced to showing that $\widehat{H}^{0}\left(G_{m, n}, A_{m}\right)$ and $\widehat{H}^{1}\left(G_{m, n}, C_{m}\right)$ are dual abelian groups. By cohomological duality, it is enough to show that for all $i \in \mathbb{Z}$,

$$
\widehat{H}^{i}\left(G_{m, n}, A_{m}\right) \cong \widehat{H}^{i}\left(G_{m, n}, B_{m}\right) .
$$

This is proved in Proposition 2.6 of [11]. We repeat the proof. Let $\mathcal{C}_{m}$ denote the $\chi$-part of the idele class group of $K_{m}$. Let $\mathcal{U}_{m}$ denote the $\chi$-part of the unit ideles of $K_{m}$. We have an exact sequence

$$
0 \rightarrow B_{m} \rightarrow \mathcal{U}_{m} / \mathrm{Cyc}_{m} \rightarrow \mathcal{C}_{m} \rightarrow A_{m} \rightarrow 0 .
$$

Since $\chi$ is not the trivial character, the $G_{m, n}$-cohomology of $\mathcal{C}_{m}$ is trivial [18, Sec. 4]. Since there is only one ramified prime in $K_{m} / K_{n}$ (the one above $p$ ), the $G_{m, n}$-cohomology of $\mathcal{U}_{m}$ is also trivial. On the other hand $\mathrm{Cyc}_{m}$ also has trivial $G_{m, n}$-cohomology, because it is a free $\mathcal{O}_{\chi}\left[G_{m}\right]$-module. We deduce that the groups $\widehat{H}^{i}\left(G_{m, n}, \mathcal{U}_{m} / \mathrm{Cyc}_{m}\right)$ are trivial. Therefore we obtain

$$
\widehat{H}^{i}\left(G_{m, n}, A_{m}\right) \cong \widehat{H}^{i+2}\left(G_{m, n}, B_{m}\right) .
$$

Since the group $G_{m, n}$ is cyclic, Tate cohomology is periodic with period 2, and we finally get (6).

For a result similar to Proposition 3, see Theorem 2 of [10].

We now want to show that $A$ and $C$ have the same characteristic ideal. In order to do that, we briefly recall the results we need from the theory of adjoints [9, Sec. 1.3], [20, Sec. 15.5]. Observe that in the literature the results are stated in the case $\mathcal{O}_{\chi}=\mathbb{Z}_{p}$, but they naturally extend to $\mathcal{O}_{\chi}$. Let $M$ be a noetherian torsion $\Lambda$-module. For all height one prime ideals $\wp$ of $\Lambda$, we denote by $\Lambda_{\wp}$ the localization of $\Lambda$ at $\wp$. Let $\psi: M \rightarrow \bigoplus_{\wp}\left(M \otimes \Lambda_{\wp}\right)$ be the natural map. We define the adjoint $\alpha(M)$ of $M$ by

$$
\alpha(M)=\operatorname{Hom}_{\mathbb{Z}_{p}}\left(\operatorname{Coker} \psi, \mathbb{Q}_{p} / \mathbb{Z}_{p}\right) .
$$

We define a $\Lambda$-module structure on $\alpha(M)$ by setting $\xi \phi(x)=\phi(\xi x)$ for $\xi \in \Lambda, \phi \in \alpha(M)$, and $x \in \operatorname{Coker} \psi$. The module $\alpha(M)$ is quasi-isomorphic to $M$, therefore

$$
\operatorname{char} M=\operatorname{char} \alpha(M) \text {. }
$$

We need an explicit formula for $\alpha(M)$. Let $\mathfrak{m}$ be the maximal ideal of $\Lambda$. Given $M$, there exists a sequence $\pi_{n}$ of nonzero elements of $\Lambda$ such that $\pi_{0} \in \mathfrak{m}, \pi_{n+1} \in \pi_{n} \mathfrak{m}$ and such that $\pi_{n}$ and char $M$ have no common zeros for all $n \in \mathbb{N}$. 
Proposition 4. Let $M$ be a noetherian torsion $\Lambda$-module. Let $\left\{\pi_{n}\right\}$ be a sequence of elements in $\Lambda$ as above. Then

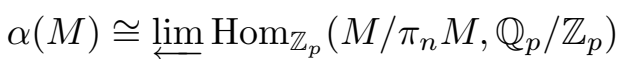

where the inverse limit is taken with respect to the morphisms induced by

$$
\begin{aligned}
& M / \pi_{n} M \rightarrow M / \pi_{m} M, \quad m \geq n \geq 0, \\
& x \bmod \pi_{n} M \rightarrow\left(\pi_{m} / \pi_{n}\right) x \bmod \pi_{m} M .
\end{aligned}
$$

We now apply Proposition 4 to $M=C$. Since $C_{n} \cong C / \omega_{n} C$ is finite for all $n \in \mathbb{N}$, it follows that $\operatorname{char} C$ and $\omega_{n}$ have no common zeros. Therefore we can take $\left\{\pi_{n}\right\}=\left\{\omega_{n}\right\}$. We obviously have $\operatorname{Hom}_{\mathbb{Z}_{p}}\left(C / \omega_{n} C, \mathbb{Q}_{p} / \mathbb{Z}_{p}\right) \cong B_{n}$. Therefore we obtain

$$
\alpha(C) \cong \lim _{\longleftarrow} B_{n}
$$

where the limit is taken with respect to the norm maps $B_{m} \rightarrow B_{n}$ with $m \geq n$. We now have

$$
\operatorname{char} C=\operatorname{char} \alpha(C)=\operatorname{char} \lim B_{n}=\operatorname{char} A .
$$

The first equality is from (7), the second by (8), the third by the main conjecture $[17,7]$ of Iwasawa theory. Since dual $\Lambda$-modules have the same Fitting ideal [14, Appendix, Prop. 3], Proposition 3 implies that

$$
\text { Fit }_{\Lambda} T(A)=\text { Fit }_{\Lambda} T(C) .
$$

Denote by $\Lambda_{n}$ the ring $\Lambda / \omega_{n} \Lambda \cong \mathcal{O}_{\chi}\left[G_{n}\right]$. We can now prove Theorem 2 of the introduction.

Proof of Theorem 2. Combining (9) with (10) and applying Theorem $1(2)$, we obtain Fit $_{\Lambda} A=$ Fit $_{\Lambda} C$. From that and from Propositions 1 and 2, we also obtain

$$
\operatorname{Fit}_{\Lambda_{n}} A_{n}=\operatorname{Fit}_{\Lambda_{n}} C_{n}
$$

But, since $C_{n}$ and $B_{n}$ are duals, by [14, Appendix, Prop. 1] we get Fit ${ }_{\Lambda_{n}} C_{n}$ $=$ Fit $_{\Lambda_{n}} B_{n}$. This concludes the proof.

Theorem 3. For all $n, \# A_{n}=\# B_{n}=\# C_{n}$.

Proof. Since obviously $\# B_{n}=\# C_{n}$, it is enough to prove that $\# A_{n}=$ $\# C_{n}$. By (9) and Theorem 1(3), we only need to show $\# T(A)_{n}=\# T(C)_{n}$. By Proposition 3, we have

$$
\begin{aligned}
\# T(C)_{n} & =\# \operatorname{Hom}\left(T(A), \mathbb{Q}_{p} / \mathbb{Z}_{p}\right)_{n}=\# \operatorname{Hom}\left(T(A)^{\Gamma^{p^{n}}}, \mathbb{Q}_{p} / \mathbb{Z}_{p}\right) \\
& =\# T(A)^{\Gamma^{p^{n}}}=\# T(A)_{n} .
\end{aligned}
$$

4. Concluding remarks. In this last section we make some observations. We keep the notations of the previous section. 
Proposition 5. The A-module $C$ is cyclic.

Pro of. Because of Proposition 2 and Nakayama's lemma applied to the local ring $\Lambda$, it is enough to prove that $C_{0}$ is a cyclic $\Lambda$-module. This is equivalent to saying that $C_{0}$ is a cyclic $\Lambda_{0} \cong \mathcal{O}_{\chi}$ module. Since $B_{0} \cong C_{0}$ as $\mathcal{O}_{\chi}$-modules, it remains to show that $B_{0}$ is cyclic over $\mathcal{O}_{\chi}$. Let $\mathcal{O}_{0}^{*}$ be the $\chi$-part of the units in the field $K$. Recall that $\mathcal{O}_{0}^{*}$ contains the free onedimensional $\mathcal{O}_{\chi}$-submodule of finite index $\mathrm{Cyc}_{0}$, and is torsion free because $\chi$ is not trivial. By the structure theorem on finitely generated modules over a principal ideal domain, $\mathcal{O}_{0}^{*}$ is a free one-dimensional $\mathcal{O}_{\chi}$-module. Therefore $B_{0}$, which is a quotient of $\mathcal{O}_{0}^{*}$, is cyclic.

By the above proposition, the structure of $C$ is determined by Fit ${ }_{\Lambda} C$. In particular, for all $n$, we have

$$
C_{n} \cong \Lambda_{n} /\left(\operatorname{Fit}_{\Lambda} C\right) \Lambda_{n} .
$$

The module $A$ is not cyclic in general, but if $A_{0}$ is cyclic then $A$ is cyclic by Nakayama's lemma, and $A_{n} \cong C_{n}$ as Galois modules for all $n \in \mathbb{N}$. In general, Theorem 2 puts some constraints on the structure of $A_{n}$. In fact, using (11) and Theorem 3 , we see that

$$
\# A_{n}=\# C_{n}=\# \Lambda_{n} / \operatorname{Fit}_{\Lambda_{n}} C_{n}=\# \Lambda_{n} / \text { Fit }_{\Lambda_{n}} A_{n} .
$$

Let $\mathfrak{m}_{n}$ be the maximal ideal of $\Lambda_{n}$. The $\Lambda_{n}$-module $M_{n}=\Lambda_{n} / \mathfrak{m}_{n} \times \Lambda_{n} / \mathfrak{m}_{n}$ has $\mathfrak{m}_{n}^{2}$ as Fitting ideal, and $\# M_{n} \neq \#\left(\Lambda_{n} /\right.$ Fit $\left._{\Lambda_{n}} M_{n}\right)$ for $n>0$. Therefore for $n>0$ we cannot have $A_{n} \cong \Lambda_{n} / \mathfrak{m}_{n} \times \Lambda_{n} / \mathfrak{m}_{n}$.

With the same techniques of Section 3 we can prove Theorem 2 in the case $p=2$ with $A_{n}$ replaced by the $\chi$-part of the narrow ideal class group of $K_{n}$ and with $B_{n}$ replaced by the $\chi$-part of the group of totally positive units of $K_{n}$ modulo the square of cyclotomic units.

It is expected that in general the $\lambda$-invariant of the Iwasawa module $A$ is zero. This question has been studied by R. Greenberg [6]. In our terminology, the condition $\lambda=0$ for the $\Lambda$-module $A$ is equivalent to $A=T(A)$. In the case $A=T(A)$, there exists an algorithm [11] to compute Fit ${ }_{\Lambda} C$.

One could try to generalize Theorem 2 to real abelian fields of prime power conductor such that $p \mid[K: \mathbb{Q}]$. Let $P$ be the $p$-Sylow subgroup of $\operatorname{Gal}(K / \mathbb{Q})$. We now have to substitute $\Lambda \cong \mathcal{O}_{\chi}[[T]]$ with $\Lambda[P] \cong \mathcal{O}_{\chi}[P][[T]]$. This is no more a local regular ring and in general there exist $\Lambda[P]$-modules which are $\mathbb{Z}_{p}$-free, but have infinite projective dimension. If one assumes an affirmative answer to Greenberg's question, that is to say, $A=T(A)$ (hence also $C=T(C)$ ), then the techniques of this paper can be adapted to prove again Theorem 2 in this situation. For a study of $\Lambda[P]$-modules, see $[8]$. 


\section{References}

[1] N. Bourbaki, Algebra I, Springer, New York, 1989.

[2] J. W. S. Cassels and A. Fröhlich, Algebraic Number Theory, Academic Press, London, 1967.

[3] P. Cornacchia, Anderson's module for cyclotomic fields of prime conductor, J. Number Theory 67 (1997), 252-276.

[4] P. Cornacchia and C. Greither, Fitting ideals of class groups of real fields of prime power conductor, J. Number Theory, to appear.

[5] M. Grandet et J.-F. Jaulent, Sur la capitulation dans une $\mathbb{Z}_{l}$-extension, J. Reine Angew. Math. 362 (1985), 213-217.

[6] R. Greenberg, On the Iwasawa invariants of totally real number fields, Amer. J. Math. 98 (1976), 263-284.

[7] C. Greither, Class groups of abelian fields, and the main conjecture, Ann. Inst. Fourier (Grenoble) 42 (1992), no. 3, 449-499.

[8] - , The structure of some minus class groups, and Chinburg's third conjecture for abelian fields, Math. Z., to appear.

[9] K. Iwasawa, On $\mathbb{Z}_{l}$-extensions of algebraic number fields, Ann. of Math. (2) 98 (1973), 246-326.

[10] J. M. Kim, S. B ae and I.-S. Lee, Cyclotomic units in $\mathbb{Z}_{p}$-extensions, Israel J. Math. 75 (1991), 161-165.

[11] J. S. Kraft and R. Schoof, Computing Iwasawa modules of real quadratic number fields, Compositio Math. 97 (1995), 135-155.

[12] L. V. Kuz'min, On formulae for the class number of real Abelian fields, Russian Acad. Sci. Izv. Math. 60 (1996), 695-761.

[13] S. Lang, Cyclotomic Fields I and II, combined 2nd ed., Grad. Texts in Math. 121, Springer, New York, 1990.

[14] B. Mazur and A. Wiles, Class fields of abelian extensions of $\mathbb{Q}$, Invent. Math. 76 (1984), 179-330.

[15] M. Ozaki, On the cyclotomic unit group and the ideal class group of a real abelian number field, J. Number Theory 64 (1997), 211-222.

[16] - On the cyclotomic unit group and the ideal class group of a real abelian number field II, ibid., 223-232.

[17] K. Rubin, The Main Conjecture, Appendix to [13].

[18] R. Schoof, The structure of the minus class groups of abelian number fields, in: Séminaire de Théorie des Nombres, Paris 1988-89, Progr. Math. 91, Birkhäuser, 1991, 185-204.

[19] W. Sinnott, On the Stickelberger ideal and the circular units of an abelian field, Invent. Math. 62 (1980), 181-234.

[20] L. C. Washington, Introduction to Cyclotomic Fields, 2nd ed., Grad. Texts in Math. 83, Springer, New York, 1997.

Corso XXV Aprile 60

14100 Asti, Italy

E-mail: cornac@dm.unipi.it 